\title{
Modelling graphene quantum dot functionalization via ethylene-dinitrobenzoyl
}

Cite as: Appl. Phys. Lett. 108, 123902 (2016); https://doi.org/10.1063/1.4944906

Submitted: 21 August 2015 . Accepted: 16 March 2016 . Published Online: 24 March 2016

Keian Noori, Hannes Hübener, (D) Emmanuel Kymakis, and Feliciano Giustino
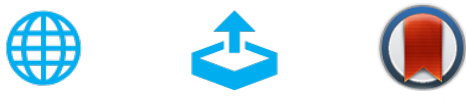

View Online

\section{ARTICLES YOU MAY BE INTERESTED IN}

Facile synthesis and photoluminescence mechanism of graphene quantum dots Journal of Applied Physics 116, 244306 (2014); https://doi.org/10.1063/1.4904958

Functionalization of graphene quantum dots by fluorine: Preparation, properties, application, and their mechanisms

Applied Physics Letters 110, 221901 (2017); https://doi.org/10.1063/1.4984238

In-situ sequential laser transfer and laser reduction of graphene oxide films Applied Physics Letters 112, 183301 (2018); https://doi.org/10.1063/1.5021862

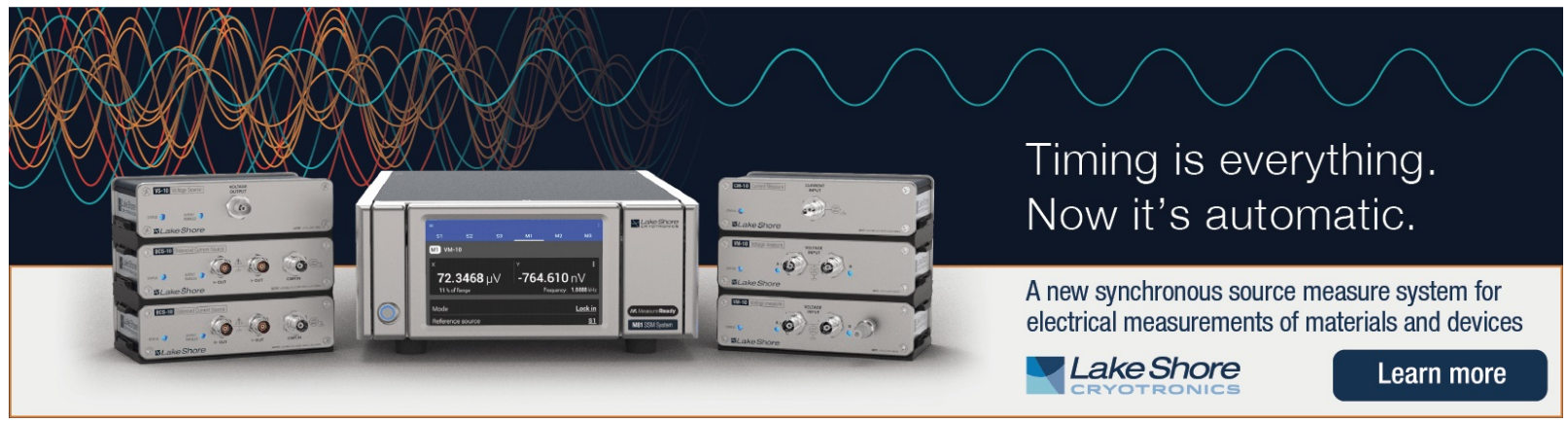




\title{
Modelling graphene quantum dot functionalization via ethylene-dinitrobenzoyl
}

\author{
Keian Noori, ${ }^{1}$ Hannes Hübener, ${ }^{1,2}$ Emmanuel Kymakis, ${ }^{3}$ and Feliciano Giustino ${ }^{1}$ \\ ${ }_{1}^{1}$ Department of Materials, University of Oxford, Parks Road, Oxford OX1 3PH, United Kingdom \\ ${ }^{2}$ Nano-Bio Spectroscopy Group and European Theoretical Spectroscopy Facility (ETSF), Universidad del País \\ Vasco CFM CSIC-UPVIEHU-MPC \& DIPC, Av. Tolosa 72, 20018 San Sebastián, Spain \\ ${ }^{3}$ Center of Materials Technology and Photonics \& Electrical Engineering Department, Technological \\ Educational Institute (TEI) of Crete, Heraklion, 71004 Crete, Greece
}

(Received 21 August 2015; accepted 16 March 2016; published online 24 March 2016)

Ethylene-dinitrobenzoyl (EDNB) linked to graphene oxide has been shown to improve the performance of graphene/polymer organic photovoltaics. Its binding conformation on graphene, however, is not yet clear, nor have its effects on work function and optical absorption been explored more generally for graphene quantum dots. In this report, we clarify the linkage of EDNB to GQDs from first principles and show that the binding of the molecule increases the work function of graphene, while simultaneously modifying its absorption in the ultraviolet region. C 2016 AIP Publishing LLC. [http://dx.doi.org/10.1063/1.4944906]

It has recently been demonstrated that the addition of ethylene-dinitrobenzoyl (EDNB) can be beneficial to the performance of organic photovoltaic (OPV) devices made from graphene oxide (GO) and poly(3-hexylthiophene) (P3HT). ${ }^{1}$ The reasons for this improvement are considered to be twofold. First, it is thought that upon linking the GO with EDNB its dispersity in the polymer matrix is increased, leading to a larger interfacial area and fewer dead-end pathways for charge transport. Second, the EDNB molecule contributes to the absorption of GO and can enhance light harvesting in the bulk heterojunction $\mathrm{GO} / \mathrm{P} 3 \mathrm{HT}$ blend.

While the linkage of the molecule to GO was identified via FT-IR measurements, ${ }^{2}$ the precise nature of the binding configuration of EDNB on graphene is not clear. Moreover, the atomistic origin of the modifications to the work function and the optical properties of graphene induced by EDNB have not been explored. Given that the EDNB attachment can be easily realized via solution processing, this could have potentially important technological benefits not just for OPV devices but also for other graphene- and graphene quantum dot (GQD)-based applications. ${ }^{3}$ In this context, a detailed knowledge of the binding configuration of EDNB on graphene as well as the induced alterations to the work function and absorption spectrum are critical. Here, we explore the impacts of EDNB on these factors from first principles density-functional theory (DFT) and time-dependent DFT (TDDFT) calculations. We first determine the likely linkage of the molecule with graphene using total energy calculations. Next, we examine the impact of the work function modification associated with the molecular linkage. Finally, using the most stable binding configuration, we examine the impact of the EDNB on the optical absorption spectrum of large graphene quantum dots.

The structural and electronic calculations were performed within the local density approximation (LDA) using the local-orbital SIESTA package, ${ }^{4}$ while the optical absorption spectra were determined within time-dependent LDA (TDLDA) using our Sternheimer TDDFT code, ${ }^{5}$ which builds on SIESTA, using the multishift method. ${ }^{6}$ For the sake of clarity, the details of the calculations are presented in the supplementary material. ${ }^{7}$

Before attempting to analyze the effect of EDNB on the work function and optical absorption of graphene, it is important to understand how the linkage between the molecule and graphene occurs and what is the most stable chemisorption configuration. In experiment, ${ }^{1}$ EDNB is linked to graphene using a two-step procedure. First, graphene is acylated by attaching $\mathrm{COCl}$ at the terminating phenyl groups. Next, EDNB is mixed with the acylated graphene, whereupon the molecule links with the acyl group via peptide bonds to form a GQD/EDNB molecule. While this linkage has been verified via FT-IR measurements, the precise nature of the bonding morphology is not known.

Our approach to clarifying this issue is to create an idealized H-terminated GQD featuring both zigzag and armchair edges. The atomic models for EDNB and our GQD are shown in Figure 1. The binding point for the EDNB (i.e., the acyl group) is placed at four different locations: the middle and corner of the zigzag edge (ZZM and ZZC, respectively), and the middle and corner of the armchair edge (ACM and ACC, respectively). These binding locations are at the phenyl edges

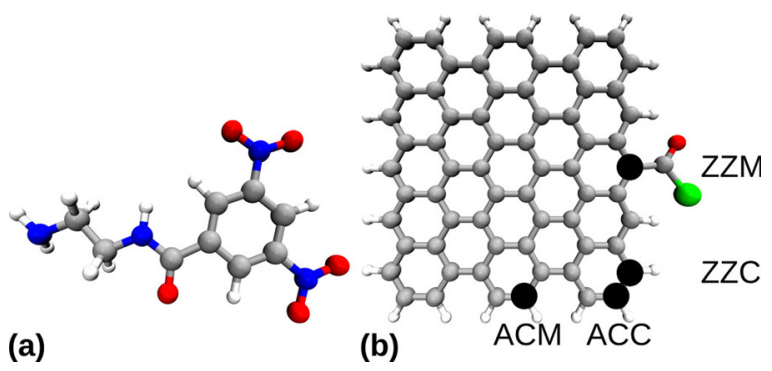

FIG. 1. The atomic structures of (a) isolated EDNB and (b) our model graphene quantum dot acylated with $\mathrm{COCl}$. The black circles indicate the four binding points for the $\mathrm{COCl}$ acyl group used to link EDNB: middle of the armchair edge (ACM) and the corresponding corner (ACC); middle of the zigzag edge (ZZM) and the corresponding corner (ZZC). The atomic color code is: white $(\mathrm{H})$, gray $(\mathrm{C})$, blue $(\mathrm{N})$, red $(\mathrm{O})$, green $(\mathrm{Cl})$. 
of the GQD and are shown in Figure 1(b). At each of these four binding sites, we attached the EDNB in four different initial configurations: the benzene ring on top of the GQD (C1), the benzene ring perpendicular to the GQD $(\mathrm{C} 2)$, the benzene ring outside of and raised above the GQD (C3), and the benzene ring outside of and coplanar to the GQD (C4). This approach generates a total of 16 unique initial configurations that allowed us to explore the majority of the conformation space for the GQD-EDNB linkage. We performed firstprinciples molecular dynamics simulations lasting over $2 \mathrm{ps,}$ showing that the EDNB tends to remain near its starting position at room temperature. This provides further justification for the choice of positional extremes for our initial configurations. These 16 initial configurations were then structurally optimized. The final structures corresponding to the ZZM C1C4 configurations are shown in Figure 2. The relative stability of each of the 16 optimized configurations was determined by calculating their respective total energies. For all four binding sites, the most stable configuration is $\mathrm{C} 1$, with a total energy $1.10,1.13,1.20$, and $1.22 \mathrm{eV}$ lower than any other configuration for the ZZM, ZZC, ACM, and ACC sites, respectively, while the least stable configuration varies with the binding site. The complete set of calculated total energies is presented in Table I. That the $\mathrm{C} 1$ configuration is always the most stable is not surprising given the stabilization of the molecule due to the physisorption of the benzene ring of the EDNB on the GQD surface (see Figure 2(a)).

The impact of EDNB on the work function of graphene was determined by examining the energy of the highest occupied molecular orbital (HOMO) for all 16 graphene-EDNB configurations, and comparing it to that of the pristine GQD. The work function was found by taking the difference between the vacuum energy and the calculated HOMO energy. A common reference potential is required in order to directly compare the work functions of the 16 different configurations. Owing to the local-orbital basis functions implemented in

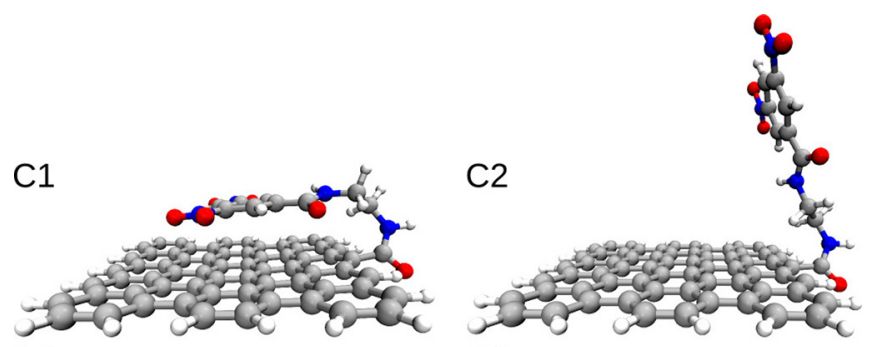

(a)

(b)

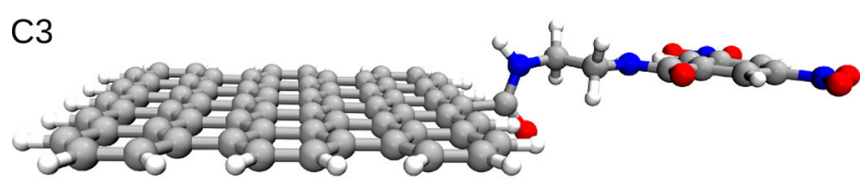

(c)

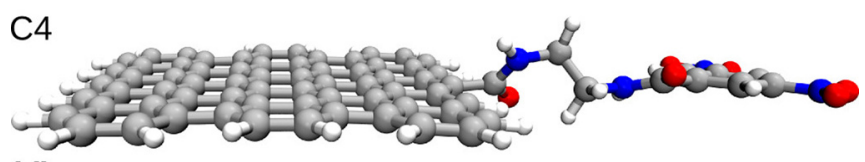

(d)

FIG. 2. Relaxed structures corresponding to the ZZM binding point. Panels (a)-(d) correspond to the initial configurations $\mathrm{C} 1, \mathrm{C} 2, \mathrm{C} 3$, and $\mathrm{C} 4$, respectively.
TABLE I. The total energy (TE) and work function $(\phi)$ for 16 GQD/EDNB configurations. The configurations $\mathrm{C} 1-\mathrm{C} 4$ refer to binding morphologies with the benezene ring of EDNB on top of the GQD, perpendicular to the GQD, outside of and raised above the GQD, and outside of and coplanar to the GQD, respectively. The locations of the EDNB linkages are noted by the four binding points ZZM, ZZC, ACM, and ACC, indicating the middle and corner of the zigzag edge, and the middle and corner of the armchair edge, respectively. The total energies are referenced relative to the most stable configuration (ZZM C1). The work function is expressed relative to that of the bare GQD. All values are in $\mathrm{eV}$.

\begin{tabular}{|c|c|c|c|c|c|c|c|c|}
\hline & \multicolumn{2}{|c|}{$\mathrm{C} 1$} & \multicolumn{2}{|c|}{$\mathrm{C} 2$} & \multicolumn{2}{|c|}{ C3 } & \multicolumn{2}{|c|}{$\mathrm{C} 4$} \\
\hline & TE & $\phi$ & $\mathrm{TE}$ & $\phi$ & TE & $\phi$ & TE & $\phi$ \\
\hline ZZM & 0.00 & 0.09 & 1.02 & 0.22 & 1.10 & 0.18 & 0.87 & 0.20 \\
\hline $\mathrm{ZZC}$ & 0.05 & 0.16 & 1.08 & 0.23 & 1.13 & 0.17 & 1.05 & 0.16 \\
\hline $\mathrm{ACM}$ & 0.05 & 0.08 & 1.20 & 0.16 & 1.13 & 0.13 & 1.20 & 0.13 \\
\hline $\mathrm{ACC}$ & 0.09 & 0.19 & 1.22 & 0.15 & 0.97 & 0.17 & 0.89 & 0.17 \\
\hline
\end{tabular}

SIESTA, we were able to use a very large computational cell for each calculation, thereby allowing the total potential to converge to zero in all directions within each cell. As a result, the vacuum energy can be taken as zero for every configuration, and the work function for each GQD/EDNB molecule is then nothing other than the absolute value of its HOMO energy. Table I reports the calculated work functions of the pristine GQD and all 16 GQD/EDNB configurations. We note that in every case the linkage of EDNB onto the GQD raises the work function. This increase is in the range of $0.09-0.23 \mathrm{eV}$ depending on the binding site and initial configuration. In the most stable configuration $(\mathrm{C} 1)$, the addition of EDNB does not significantly impact the work function $(<0.1 \mathrm{eV})$ in the middle of a GQD edge (ZZM, ACM), while it has a more pronounced effect $(>0.15 \mathrm{eV})$ at a GQD corner (ZZC, ACC). The increase in the work function of the functionalized GQD results from two effects: (i) the quantum mechanical coupling of the EDNB and GQD states, which leads to a lowering of the HOMO as compared to the bare GQD; and (ii) the presence of the EDNB causes a redistribution of charge in the ground state of the combined GQD/EDNB molecule, which leads to the formation of an electrostatic dipole that acts to increase work function of the molecule. Figure 3 shows the redistribution for the ZZM C1 configuration.

The linkage of EDNB to the phenyl edges of GQDs represents one possibility for tuning their optical absorption, arising from the broadening of the absorption spectra due to the presence of nitro groups. ${ }^{8}$ In order to explore the effect of EDNB on the absorption spectrum of realistic GQDs, we move away from our idealized model and consider a realistic nanographene (NG) structure. NGs have been demonstrated to be useful for LED and solar cell applications, amongst others (see Ref. 9 and references therein), and here, we examine the impact of the EDNB linkage on the absorption spectrum of the large NG hexa-peri-hexabenzocircumcoronene (HBCC,5c). ${ }^{9}$ In our model, EDNB is linked to a phenyl edge of HBCC 5,5c in the place normally occupied by one of the butane groups, and in the most stable $\mathrm{C} 1$ configuration. Both the experimental structure of $\mathrm{HBCC} 5,5 \mathrm{c}$ and the EDNB-functionalized molecule are shown in Figure 4. The calculated spectrum of HBCC5,5c is shown in Figure 5 and features two main peaks: one sharp peak is present at $2.3 \mathrm{eV}$, 

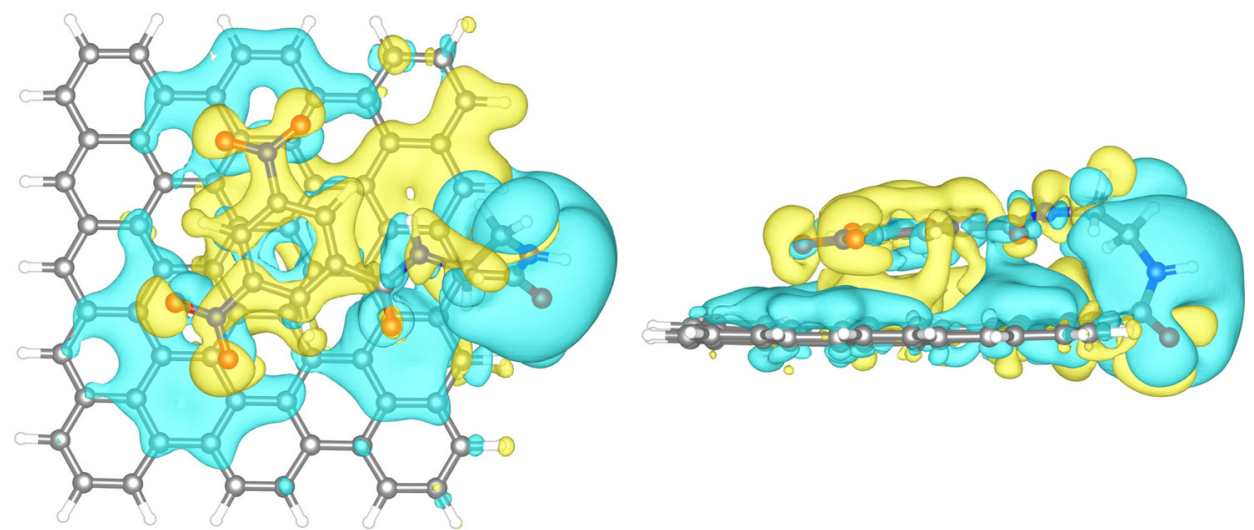

(a)

(b)

FIG. 3. The charge redistribution in the $\mathrm{ZZC} \mathrm{C1}$ configuration from (a) the top and (b) the side. This redistribution is determined as $\Delta n=n_{\text {molecule }}-n_{\mathrm{GQD}}-n_{\mathrm{EDNB}}-n_{2 \mathrm{H}}$. Here, $n_{\text {molecule }}$ represents the charge density of the GQD/EDNB molecule, while $n_{\mathrm{GQD}}$ and $n_{\mathrm{EDNB}}$ represent the charge densities of the isolated GQD and EDNB, respectively, frozen in their configuration from the interface. In both cases, the dangling bonds resulting from the forced separation of EDNB from the GQD are terminated with a $\mathrm{H}$ atom, which is reflected in the charge redistribution balance by the $n_{2 \mathrm{H}}$ term. The yellow isosurface indicates local electron accumulation, while the blue isosurface indicates local electron depletion.

while a broader feature exists from 4.6 to $5.0 \mathrm{eV}$. There is an additional small peak at $3.4 \mathrm{~V}$. The experimental spectrum from Ref. 9 is also shown for comparison. The calculated peaks are redshifted with respect to the experimental spectrum by approximately $0.5 \mathrm{eV}$, but otherwise the agreement with experiment is satisfactory. We tentatively assign the redshift in our calculations to shortcomings in the theoretical description of the optical absorption process that are not captured by TDLDA calculations in vacuum ${ }^{10}$ (e.g., vibronic and solvation effects). In any case, we note that the calculations are in line with experimental measurements, and stress that the aim of this work is not to precisely reproduce the experimental spectrum of $\mathrm{HBCC} 5,5 \mathrm{c}$, but rather to assess the impact on the absorption spectrum of GQDs upon linkage of EDNB. This effect can be seen in Figure 5. All peaks show a small $(<0.1 \mathrm{eV})$ redshift, which may be the result of the underlying structural change of the NG after linkage with

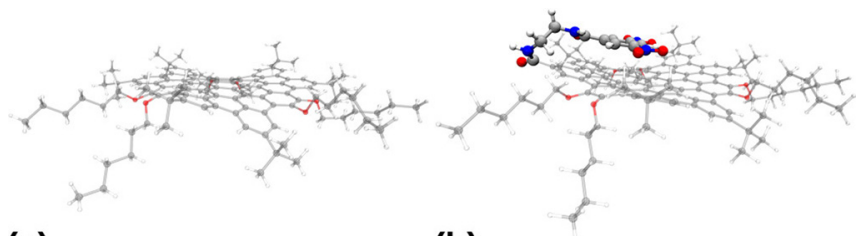

(a)

(b)

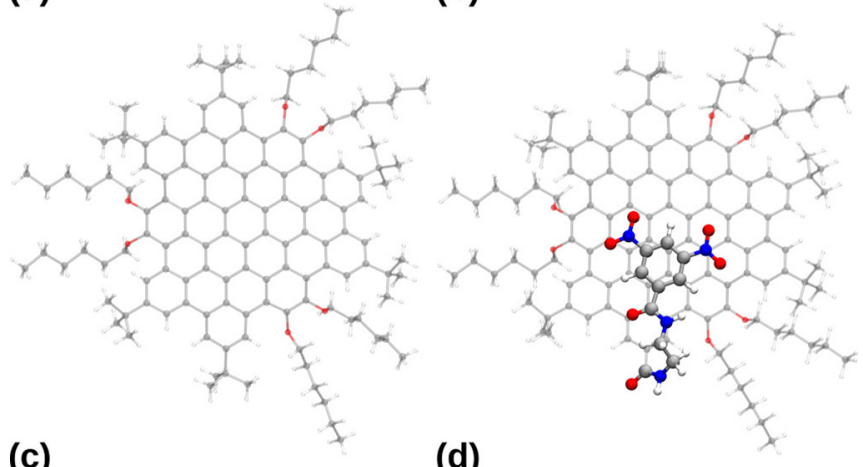

(c)

(d)

FIG. 4. The atomic structure of the HBCC,5c molecule without ((a) and (c)) and with ((b) and (d)) EDNB. The atomic color code is identical to that of Figure 2.
EDNB, and is consistent with the redshift observed in the visible spectrum for graphene oxide/EDNB solar cells. ${ }^{1}$

The effect of EDNB on the absorption of HBCC5,5c can be estimated by determining the increase in the number of absorbed photons before and after the linkage of the small molecule. Our calculations show a slight increase in absorbed photons after functionalization of HBCC5,c with EDNB. The origin of this increase was investigated by examining the TDDFT density response to the perturbation at the peaks. For the optical transitions at 2.3 and $3.4 \mathrm{eV}$, the density response is localized on the HBCC5,5c for all three light polarizations, indicating that these transitions result only from the NG. This result is unsurprising upon examination of the calculated spectrum of EDNB (see inset of Figure 5). EDNB displays weak absorption below $4 \mathrm{eV}$, with the main peak at $4.6 \mathrm{eV}$. Analysis

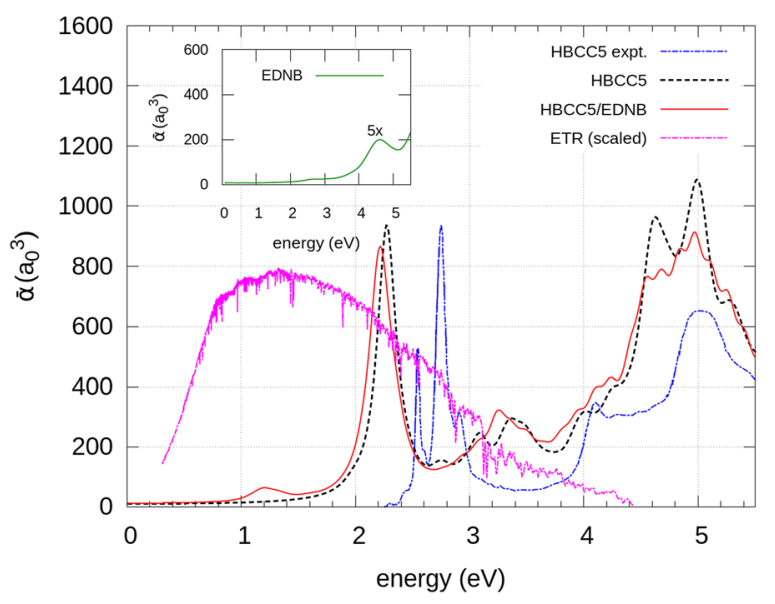

FIG. 5. The calculated absorption spectra of the HBCC,5c molecule with (solid red) and without (dashed black) EDNB. The experimental HBCC,5c spectrum from Ref. 9 (dash-and-dot blue) is also shown for comparison. The calculated spectrum of EDNB (magnified by 5 times) is shown in the inset. The extraterrestrial (ETR) solar spectrum (dash-and-dot purple) is also presented to show its overlap with the calculated and experimental data; note that this spectrum has been arbitrarily scaled in order to match the other spectra. The calculated spectra are represented as the isotropic averages of their respective polarizability tensors (in units of bohr ${ }^{3}$ ), while the experimental spectrum is scaled to match the calculated peak at $2.3 \mathrm{eV}$. 
of the small peak at $1.2 \mathrm{eV}$ in our spectrum reveals that this transition comes from the sidechains of the HBCC5,5c molecule. It is therefore to be expected that the effect of EDNB on the electronic structure of $\mathrm{HBCC} 5,5 \mathrm{c}$ will be negligible below $4 \mathrm{eV}$ and that the observed increase in absorption results from the associated structural modification of the molecule induced by the linkage of EDNB.

For the feature at $4.6-5.0 \mathrm{eV}$, however, the density response is coupled between the NG and EDNB, which demonstrates an intra-molecular transition (see Figure $\mathrm{S}^{7}$ ). Interestingly, the density response of this transition derives almost exclusively from the perturbation polarized perpendicularly to the basal plane of $\mathrm{HBCC}, 5 \mathrm{c}$ and the benzene ring of EDNB, which suggests that the $\mathrm{C} 1$ configuration of EDNB is advantageous for modifying the absorption spectrum of GQDs. By comparing the density response of this transition and the wavefunctions of the band extrema of the HBCC5,5c/ EDNB molecule, we deduce that this peak in the spectrum arises from a HOMO-LUMO transition. These observations indicate that the binding of EDNB to HBCC5,5c enhances its absorption in the high energy range above $4 \mathrm{eV}$.

Our calculations suggest that the redshift observed in the experiments upon functionalizing graphene with EDNB is not due to a modification of the electronic structure of reduced graphene oxide (rGO). Instead, the experimental shift may be explained by assuming that, by increasing the solubility of rGO, EDNB slightly modifies the packing density of rGO and therefore the magnitude of solvation effects. Such effects would not be captured by our calculations, which are performed for graphene-EDNB in vacuum.

In conclusion, we have shown that both the work function and optical spectrum of graphene quantum dots and similar graphene molecules can be modified by the linking of EDNB to the phenyl edges via amine groups. The most stable binding morphology consists of the benzene ring of the EDNB lying on top of the basal plane of graphene. Our calculations also indicate that EDNB causes an increase in the work function of graphene up to $0.2 \mathrm{eV}$, allowing for the possibility of energy-level tuning. Using TDDFT, we have computed the optical absorption of a large nanographene and have demonstrated that the linkage of EDNB to this molecule causes a redshift in its spectrum of $\sim 10 \mathrm{~nm}$, which is smaller than but in the same direction as experiment. This shift is responsible for a slight increase in the number of photons absorbed, resulting from electronic structure modification in the UV region and structural changes at lower energy. The additional redshift seen in experiment may be the result of modified solvation effects.
Combined with its ability to increase the dispersity of graphene in polymer matrices, the energy-level tuning and optical absorption broadening induced by the linkage of EDNB indicate that this molecule should be beneficial in graphene quantum dot-based optoelectronics.

The authors would like to thank G. Volonakis for insightful discussions regarding the work function modification in graphene. The research leading to these results has received funding from the European Union Seventh Framework Programme under Grant Agreement No. 604391 Graphene Flagship. This work was supported by the European Research Council (EU FP7/ERC Grant No. 239578), the UK Engineering and Physical Sciences Research Council (Grant No. EP/J009857/1), and the Leverhulme Trust (Grant No. RL-2012-001). H.H. acknowledges support from the People Programme (Marie Curie Actions) of the European Union's Seventh Framework Programme FP7-PEOPLE2013-IEF Project No. 622934. Calculations were performed at the University of Oxford Advanced Research Computing facility. Figures involving atomic structures were rendered using VMD. ${ }^{11}$ The charge redistribution in Figure 3 was produced using VESTA. ${ }^{12}$

${ }^{1}$ M. M. Stylianakis, G. D. Spyropoulos, E. Stratakis, and E. Kymakis, Carbon 50, 5554 (2012).

${ }^{2}$ M. M. Stylianakis, M. Sygletou, K. Savva, G. Kakavelakis, E. Kymakis, and E. Stratakis, Adv. Opt. Mater. 3, 658 (2015).

${ }^{3}$ A. C. Ferrari, F. Bonaccorso, V. Fal'ko, K. S. Novoselov, S. Roche, P. Boggild, S. Borini, F. H. L. Koppens, V. Palermo, N. Pugno, J. A. Garrido, R. Sordan, A. Bianco, L. Ballerini, M. Prato, E. Lidorikis, J. Kivioja, C. Marinelli, T. Ryhanen, A. Morpurgo, J. N. Coleman, V. Nicolosi, L. Colombo, A. Fert, M. Garcia-Hernandez, A. Bachtold, G. F. Schneider, F. Guinea, C. Dekker, M. Barbone, Z. Sun, C. Galiotis, A. N. Grigorenko, G. Konstantatos, A. Kis, M. Katsnelson, L. Vandersypen, A. Loiseau, V. Morandi, D. Neumaier, E. Treossi, V. Pellegrini, M. Polini, A. Tredicucci, G. M. Williams, B. Hee Hong, J.-H. Ahn, J. Min Kim, H. Zirath, B. J. van Wees, H. van der Zant, L. Occhipinti, A. Di Matteo, I. A. Kinloch, T. Seyller, E. Quesnel, X. Feng, K. Teo, N. Rupesinghe, P. Hakonen, S. R. T. Neil, Q. Tannock, T. Lofwander, and J. Kinaret, Nanoscale 7, 4598 (2015).

${ }^{4}$ J. M. Soler, E. Artacho, J. D. Gale, A. García, J. Junquera, P. Ordejón, and D. Sánchez-Portal, J. Phys.: Condens. Matter 14, 2745 (2002).

${ }^{5}$ H. Hübener and F. Giustino, Phys. Rev. B 89, 085129 (2014).

${ }^{6}$ H. Hübener and F. Giustino, J. Chem. Phys. 141, 044117 (2014).

${ }^{7}$ See supplementary material at http://dx.doi.org/10.1063/1.4944906 for details of the computational methods as well a visualization of the density response of the HBCC5,5c/EDNB molecule.

${ }^{8}$ Y. He, X. Wang, J. Zhang, and Y. Li, Macromol. Rapid Commun. 30, 45 (2009).

${ }^{9}$ Q. Zhang, H. Peng, G. Zhang, Q. Lu, J. Chang, Y. Dong, X. Shi, and J. Wei, J. Am. Chem. Soc. 136, 5057 (2014).

${ }^{10}$ A. Zangwill and P. Soven, Phys. Rev. Lett. 45, 204 (1980).

${ }^{11}$ W. Humphrey, A. Dalke, and K. Schulten, J. Mol. Graphics 14, 33 (1996).

${ }^{12}$ K. Momma and F. Izumi, J. Appl. Crystallogr. 44, 1272 (2011). 